\title{
Improving the yield of 'Chok Anan' (MA 224) mango with potassium nitrate foliar sprays
}

\begin{abstract}
Unsynchronized flowering behavior and low fruit set are phenomenon that resulted in low production of mango fruit. The effects of a chemical inducing substance, potassium nitrate (KNO3), on enhancement of flowering on the mango clone óchok Ananô (MA 224) and, ultimately, the fruit production were studied. Initially, 12-month-old and 5-year-old mango trees were sprayed with $1 \%, 2 \%$, and 5\% KNO3. The 5-year-old mango trees showed earlier flowering and higher fruit set with $2 \% \mathrm{KNO} 3$ followed by $5 \%$ and $1 \% \mathrm{KNO}$. However, only $1 \% \mathrm{KNO} 3$ initiated flowering on young mango trees. In conclusion, the flowering response to $\mathrm{KNO} 3$ depends on the age of tree.
\end{abstract}

Keyword: Mangifera indica; Low production; óchok Ananô clone; Potassium nitrate; Flowering 